\title{
OPTICAL SIGNATURES OF BURIED MINES
}

\author{
Charles A. Hibbitts (karl.hibbitts@jhuapl.edu) \\ Johns Hopkins University Applied Physics Lab, 11100 Johns Hopkins Rd., \\ Laurel, Md. 20723
}

\begin{abstract}
Soils can be disturbed by the burial of mines. When disturbed, the soils have a smaller effective size distribution than when undisturbed and this difference can be detected optically. Apparent emissivity differences in the thermal infrared is an effective passive optical technique developed by others for discriminating disturbed silicate soils from undisturbed soils and appears particularly sensitive to the presence of grains less than about $60 \mu \mathrm{m}$ in diameter. It is not, however, an effective means for detecting grain size variations in carbonate sands. Optical techniques that may prove less sensitive to composition include 1) Changes in the reflectance in the shortwave infrared outside of absorption features nominally near 2.4 $\mu \mathrm{m}$, and 2) Reflectance changes due to photometric backscattering by particulate surfaces. The reflectance of particulate surfaces increases with decreasing phase angle, peaking sharply at opposition when the illumination and detector are collocated $\left(0^{\circ}\right.$ phase angle). For a given high-albedo material, finer-grains are brighter than larger-grained powders when observed at opposition.
\end{abstract}

Key words: Remote Sensing, Spectroscopy, Photometry, Landmines, IED, LTEX

\section{Introduction}

Detecting and countering Improvised Explosive Devices (IEDs) is a central theme to US and other NATO countries operating in Iraq and Afghanistan. The US spent over USD3 billion in 2006 on countermeasures to IEDs. The threat is greater than the US first estimated, often with highly educated persons building the weapons with others delivering them. The costs of IEDs are low, but countermeasures can be both expensive and not fully effective. The US is pursuing many levels of attacks to counter IEDs, considering it a huge long lasting problem that will not lend itself to a single solution. Tactics range from direct detection and electronic countermeasures to going after the bomb makers, suppliers, and the bombers, as well as in some parts of the world, attempting to apply crisis prevention to provide people incentives to not resort to terrorism. IEDs are employed and detonated by a variety of means. Some of the most notorious are vehicle-born IEDs, but other common strategies are burying them in roads, hiding them alongside roads disguised as 
trash, or even hanging them from bridges. They can be detonated remotely by cell phones or command wire, as well as by suicide bombers. Burying IEDs on or alongside roads continues to be a common method of emplacement. The act of burying the mine will leaves traces invisible to the eye that can be detected via optical remote sensing. The burial of mines will disturb soil so that it has a different grain size distribution and possibly packing density than surrounding undisturbed soils. The grain size of the disturbed soil will usually be smaller and the packing density lower. These differences will persist until the surface is reprocessed through the washing and sorting by rain and wind (Johnson et al., 1998). The rate of weathering the surface to reset the soil conditions would be dependent on the environment, with desert surfaces potentially maintaining differences for several months (Horton et al., 1998). However, in a wet environment such as a beach, the reset time can be virtually instantaneous and in a dusty environment such as next to a well-traveled unimproved surface it may take only days or hours to cover the ground with a masking layer of road dust and loess. While the quick reset times in the later examples may pose limitations for inferring the presence of buried IEDs, a technique sensitive to a change in dust abundance or distribution could also be used to detect other human activity related to IED emplacement. The ability to infer the presence of buried IEDs could be a rapid process covering a large area as a tool for an area reconnaissance. Possible IED sites could then be investigated with slower, more resource intensive, but definitive methods.

\section{Infrared spectroscopic techniques for detecting disturbed soils}

All surfaces reflect light and emit thermal energy. The intensity, distribution along the electromagnetic spectrum, and polarization can all be measured and each provides information on surface composition and structure. Our eyes are excellent detectors of relative intensity, but are poor at detecting the polarization of light and are sensitive to light only over a very narrow wavelength range where solar illumination is greatest from about $400 \mathrm{~nm}$ to $700 \mathrm{~nm}$. Thus, our eyes provide excellent context information for object identification but limited information on the composition or micro-physical structure of objects. The color we perceive for many geologic materials is largely determined by highly energetic electronic transitions within transition metals (crystal-field transitions), or transfers of charge between cations. Only a small fraction of atoms need to be transition metals to imbue color and are not necessarily representative of the bulk composition. For instance, the coloring of amethyst and rose quartz are due to trace amounts of iron and titanium, respectively. When these constituents are absent, many dissimilar rock-forming minerals can have the same color (or be colorless) at visible 

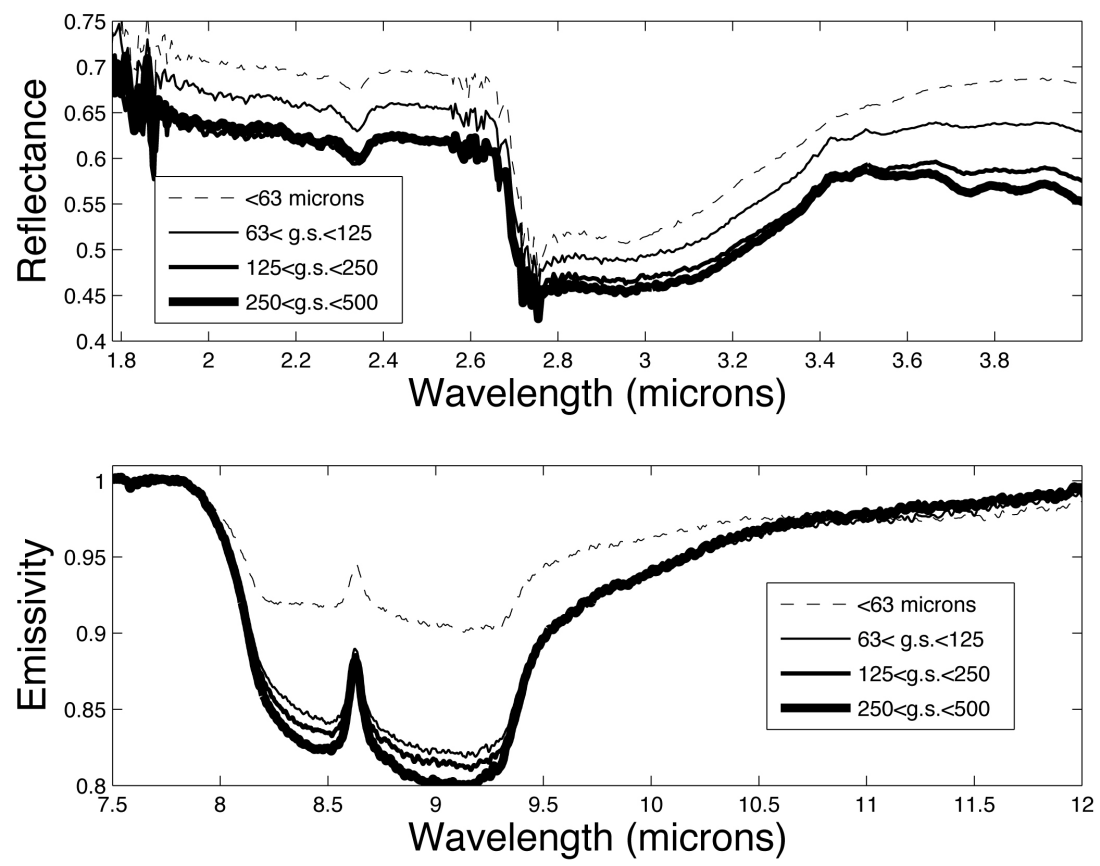

Figure 1. Directional hemispherical reflectance of ground and wet-sieved sand from San Onofre Beach, Ca. (a) The NIR and SWIR reflectance increases for the grain size fractions < 250 microns compared to larger size fractions. (b) The calculated emissivity of same sample assuming Kirchhoffs Law (emissivity $=1$ - reflectance). The emissivity at the bottom of the reststrahlen bands decrease with decreasing grain size, especially for the smallest grain size fraction $<63$ microns.

wavelengths: such as salts, carbonates, as well as quartz. Thus, discerning the composition of the earths surface using optical remote sensing has largely been the realm of lower energy, longer-wavelength infrared spectroscopy.

The brightness and spectral shape of a particulate surface is fundamentally controlled by both its ability to scatter, reflect, and absorb incident and previously scattered illumination back to the observer. The absorption of light changes with wavelength for most materials while the scattering does less so, at least in the visible to near infrared. For instance, both snow and sand are bright at visible wavelengths because they scatter the sunlight without absorbing much. Both will also color light blue that travels great lengths (several feet). However, they appear very different in the SWIR because of different absorbing and scattering behaviors at those energies. Geologic materials also tend vary in their brightness at infrared wavelengths. These variations are due to changes in both the scattering and absorption characteristics of the 
material and are caused by vibrations within the molecules and by lowerenergy electronic transitions. Thus, this short-wave infrared (SWIR) region of $1-2.5 \mu \mathrm{m}$ has been exploited for decades for determining the composition of surfaces and individual minerals (e.g. Adams, 1975). However, not all minerals possess electronic transitions or bonds that vibrate at SWIR wavelengths. Those that do not, such as quartz and some other common rock forming minerals are spectrally bland in the SWIR. However, these minerals tend to be very spectrally active at longer wavelengths, most notably in the 8-12- $\mu \mathrm{m}$ atmospheric window (e.g. Estep-Barnes, 1977; Salisbury, 1987). Commonly termed the thermal infrared (TIR) because thermally emitted radiation far outshines any reflected solar illumination, spectral variations at these wavelengths are controlled by vibrations in the structure of the molecule. Mineral classes of silicates are readily discerned at these wavelengths, and because atomic substations for the silica and the presence of charge-compensating ions alter the crystal structure, the frequency of vibrations are changed which shift spectral features and create new ones. Because of the significant absorption of light in both the SWIR and TIR, compared with the visible, scattering by particulate media can have a significantly different effect on the spectral distribution of energy at those wavelengths. At visible and near infrared (NIR) wavelengths where many minerals are not strongly absorbing, a wide range of particle sizes will be nearly equivalently bright because scattering will eventually lead to the light exiting the material without much energy being absorbed. At slightly longer SWIR wavelengths materials are more, but often only slightly absorbing, and particulate materials remain bright. However, the absorption is also usually sufficient so that finer-grained particulates that scatter more light will appear brighter than larger-grained particles (Okin and Painter, 2004). When more absorbing, such as within absorption features, a relatively small amount of light will be scattered out of the surface except for the finest grain sizes and the material will remain dark. At TIR wavelengths grain size difference manifest different, less intuitive effects because the illumination is both emitted and subsequently reflected and scattered by itself. Most materials are also highly absorbing at these wavelengths, and at some wavelengths for some materials, they are so absorbing that they become semi-metallic, opaque but reflective i.e. like metal at visible wavelengths.

The reststrahlen features of silicate minerals at $8-10 \mu \mathrm{m}$ are an example of this semi-metallic behavior. These bands are sensitive to variations in grain size as well as compositional variations (e.g. Salisbury and Wald, 1992; Johnson et al., 1998). The TIR spectra of finer-grained silicate minerals have shallower reststrahlen bands (become less reflective) and exhibit lower contrast than do larger-grains characteristic of undisturbed soils. The lower reflection becomes significant when grain sizes are sufficiently small so that 

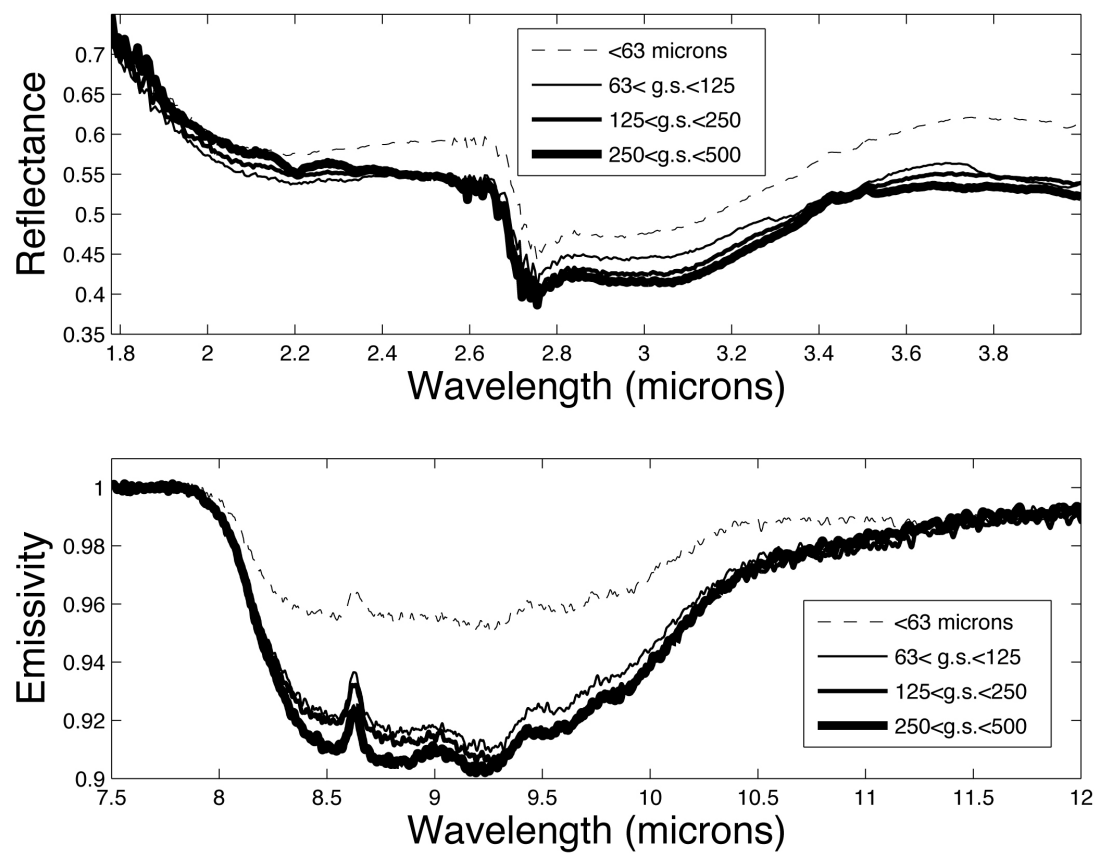

Figure 2. (a) Directional hemispherical reflectance and (b) emissivity of ground and wet-sieved mixture of fine-grained fragments of various siliceous rocks collected from desert pavements near Mecca Hills, Ca. Plots are of same style as Figure 1.

reflection is less efficient and the particles can begin to absorb and scatter the infrared radiation (Johnson et al., 1998). Porosity effects and consequential photon trapping do not appear to be a significant factor for irregularly shaped grains such as quartz. However, it remains possible that the reststrahlen bands of plate-like mineral grains such as phyllosilicates would be dependent on porosity and packing. A tightly packed surface of flat grains will have fewer voids and possess grains oriented with the long axis horizontal to reflect light with fewer bounces. A porous surface of randomly oriented grains would, in contrast, tend to reflect light into itself so that a sufficiently porous surface will contain enough multiple reflections to erase spectral contrast (e.g. Conel, 1969).

\subsection{LABORATORY SPECTRAL RESULTS AND DISCUSSION}

The SWIR of quartz-rich sand (Figure 1a) and fine-grained debris of desert silicate rocks (Figure 2a) are largely bland. These spectra are of samples 
that have been wet-sieved into various size fractions and subsequently ovendried for several hours at about $100^{\circ} \mathrm{C}$. Multiple scattering leads to increased reflectance of the finer size fractions. There is a small amount of clay present that causes the $2.2-2.3-\mu \mathrm{m}$ bands. The large, broad absorption feature near $3 \mu \mathrm{m}$ is due to the presence of the $\mathrm{OH}$ - cation vibration in the clay or perhaps in another common alteration product of silicates such as goethite $(\mathrm{FeOOH})$. In Figure 1a, the increase in reflectance for the 125 to $250-\mu \mathrm{m}$ size fraction beyond $3.5 \mu \mathrm{m}$, suggests that scattering begins to brighten quartz grains whose diameters are approximately ; $50 \mathrm{x}$ the wavelength of light. This effect is not obvious in the spectra of the compositionally more diverse sample in Figure $2 \mathrm{a}$ and only the finest grain size fraction significantly brightens. At the short wavelengths for both samples, where the signal-to-noise of the measurements begin to degrade, the reflectances of all grain sizes increase and begin to converge as scattering dominates with less absorption. Differences in grain sizes affect the SWIR reflectance spectra of carbonate sand much more (Figure 3a). The spectral structure is largely due to the carbonate and possibly organic contaminants. As with silicate sand, the reflectance of all size fractions of carbonate sand increase and merge at shorter wavelengths. The depth of weak absorption bands (such as at 2.3 and $2.5 \mu \mathrm{m}$ ) increases with increasing grain size whereas the reflectance in strongly absorbing regions (about $3.4 \mu \mathrm{m}$ ) only increases for the smallest size fraction when scattering becomes relevant.

The thermal infrared spectra of the two silicate samples are consistent with the results of Johnson et al., (1998), specifically that the reststrahlen band depth decreases with decreasing grain size, becoming significantly shallower only for the smallest size fraction (Figures $1 b$ and $2 b$ ). The emission spectra of the beach sand exhibit the reststrahlen doublet split by the higher emissivity Christensen feature of quartz. The emission spectra of the ground desert rock debris in Figure $2 b$ possess these features, though subdued and affected by other silicate features that broaden the long-wavelength reststrahlen band. This spectral contamination is consistent with the observed presence of dark mafic minerals in the desert sample. In contrast to the emission spectra of silicates, the emission spectra of carbonate sands do not change consistently with grain size (Figure $3 b$ ). The depth of the strong carbonate band near 11.3 $\mu \mathrm{m}$ does not change significantly although for the finest size fraction, it does shift position and change shape. Also, bands appear in the 8-10 $\mu \mathrm{m}$ silicate region. The later effect could be due to the presence of silicates in the finest size fraction and the changes in the $11.3-\mu \mathrm{m}$ band are likely due to a spectral asymmetry in the loss of the carbonate sands semi-metallic reflectance in this band. 

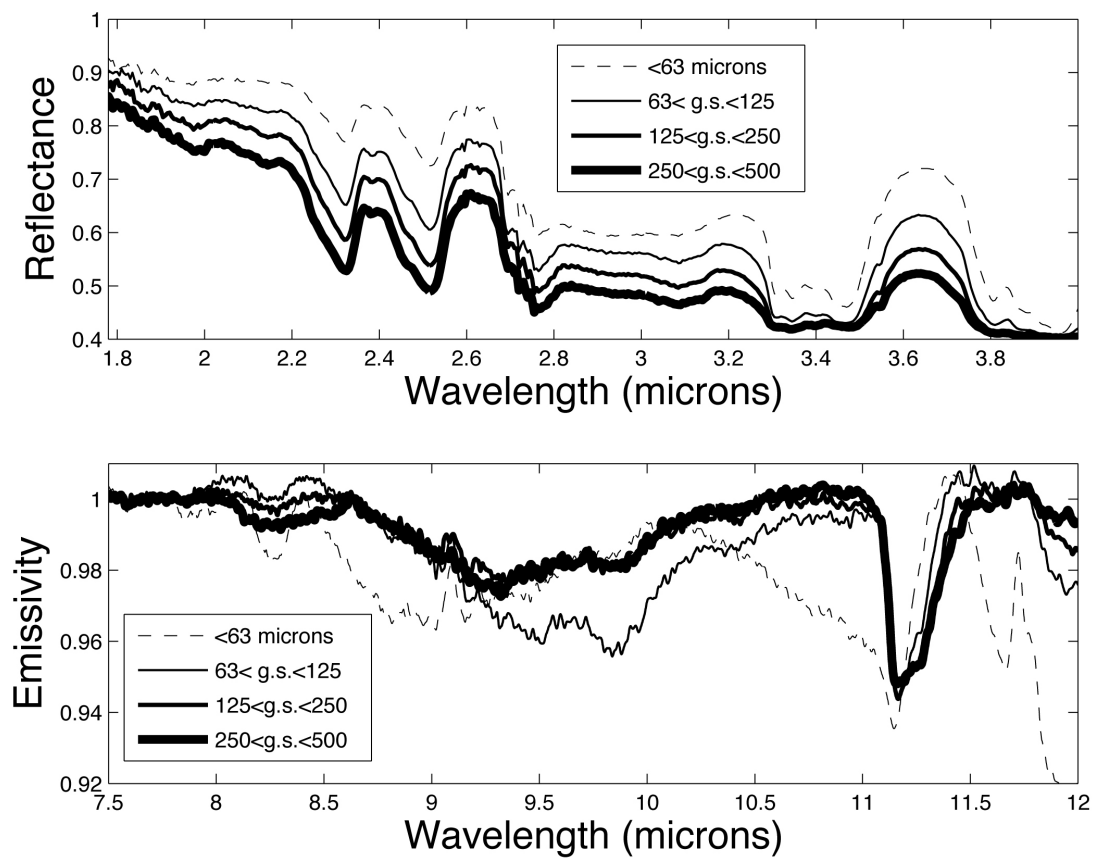

Figure 3. (a) Directional hemispherical reflectance and (b) emissivity of ground and wet-sieved carbonate sand. The increase reflectance with decreasing grain size is present over a wider range of particle sizes than for siliceous material. The thermal emission does not change progressively with grain size. The $11.3-\mu \mathrm{m}$ feature is due to the carbonate. The structure between about 8.5 and $10.5 \mu \mathrm{m}$ may be due to some silicates.

\section{Backscattered illumination effects for detecting disturbed soils}

The backscattering of light by particulate materials results in enhancement and polarization of the reflected illumination at phase angles near $0^{\circ}$. Multiple scattering of light between grains induces sharply increasing brightness below $1^{o}$ phase angle due to the constructive interference of light between portions of wave fronts traveling in opposite directions along the same multiple scattering path (Nelson et al., 1998). This is sometimes referred to as the weak localization of light or coherent backscattering and results in an increase in reflected illumination at opposition that is significant, though less than the surge associated with single scattering. For instance, high-albedo 1 to $10 \mu \mathrm{m}$ diameter dust manifests as a surge at opposition of approximately 40 percent or greater increase in brightness than when viewed at $10^{\circ}$ phase (Buratti et al., 1998). As the grain size increases or albedo decreases, the illumination surge at opposition decreases. However, as the albedo becomes exceedingly low 


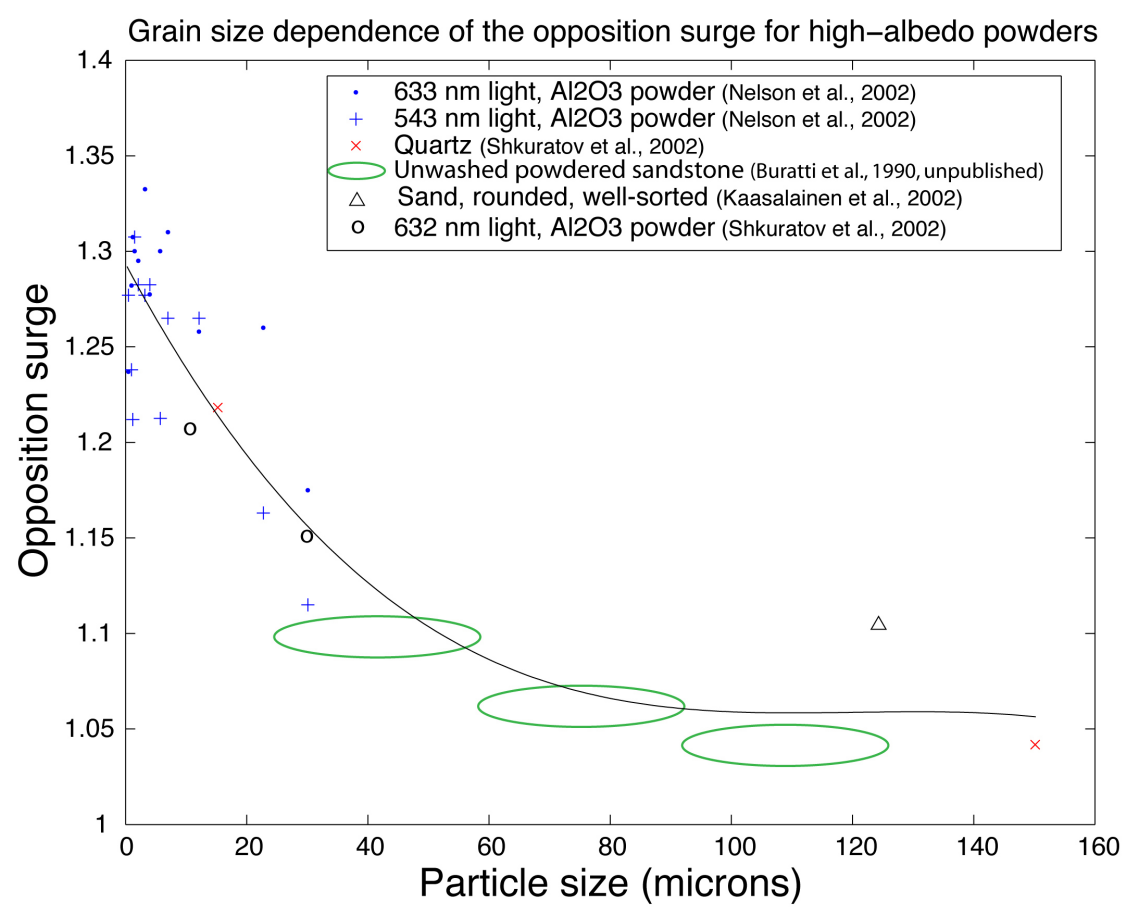

Figure 4. Grain-size dependence of the photometric brightening of high-albedo particulate materials. The surge is a ratio of the sample brightness near $0^{\circ}$ phase to that at approximately 5 or $10^{\circ}$ phase. Finer-grained, high-albedo particulate materials have the largest nonlinear increase in illumination.

so that single scattering dominates, the surge increases again (Shkurotov et al., 2002). Dark granular material has different backscattering characteristics dominated by single-scattering phenomena where grains are hidden by other grains and are not well illuminated by scattered light. The angular width of this geometric opposition surge peak is wider than for high-albedo materials and is greater, capable of brightening by a factor of up to two (Hapke, 1986).

The increase in reflectance due to backscattering at very low phase angles is dependent on grain size, as well as albedo, and offers an optical technique independent of spectral measurements for inferring grain size. For an increase in reflectance due to backscattering, the wavelength of light measured needs to be several times the size of the particle (Shkuratov et al., 2002), the optimum phase angle is less than a few tenths of a degree, and the incident illumination needs to be collimated to a similar divergence. Illumination enhancement is greatest at $0^{\circ}$ phase angle. Attempts to model the photometric backscatter effect of particulate media have ranged from theo- 
retically complex solutions to the scattering phenomenon (Mishchenko et al., 2000), to numerical solutions based upon ray tracing (Muinonen and Sarrinen, 2000), and a semi-quantitative analytical simplification of the scattering model (Shkurotov et al., 1999). For very dark materials, the optical constant which describes absorption, the imaginary portion of the complex index of refraction, $\mathrm{k}$, is sufficiently high that the material is single-scattering. However, the absorption coefficient of most geological materials is sufficiently low in the visible and NIR that multiple scattering dominates as seen in the figures of the previous section.

\subsection{EXPERIMENTAL RESULTS AND DISCUSSION}

The opposition surge of particulate materials is greater for fine-grained materials than for large grained materials in high-albedo materials with no evidence for a compositional dependency (Figure 4). Each data point in Figure 4 is a ratio of the brightness at near $0^{o}$ phase to the brightness at a phase angle of at least $4^{\circ}$, and thus beyond the angle to which coherent backscattering is a factor. The solid line is a multi-order polynomial fit to each point, or center of mass of the region for the unwashed sandstone. The dimensions of the ovals are defined by the 1-sigma deviation of those measurements. All ratios are derived from laboratory measurements conducted by others of high-albedo materials that are well-sorted, with the exception of the powdered sandstone that is red, lower-albedo, and unwashed (and thus grains will be coated with fine particulates). That the surge of the unwashed powdered sandstone is consistent with a surge expected from washed but higher-albedo particles suggests that because the sandstone is darker (redder) than the white powders used in determining the other opposition surge data, a given sieve size of ground sandstone will exhibit a smaller surge than the same sieve size of clean, higher-albedo albedo material. The overlap with the surge of higheralbedo materials demonstrates the possible need to independently separate albedo effects from grain size effects in using this technique to estimate grain size. Figure 4 also shows that high-albedo particles of the size $(10-20 \mu \mathrm{m})$ found coating larger grains in disturbed soils (Johnson et al., 1998) would be expected to have an opposition surge of approximately 20 to $25 \%$ compared to the background material that would have a surge of no more than $5 \%$.

Preliminary field measurements were made of holes dug and refilled in an unimproved road surface in the wet temperate coastal region of Washington Sate to simulate IEDs buried in other than desert soil. The road is of moderately low albedo material, darker than powdered quartz or aluminum oxide and is frequented on an hourly or daily basis, largely by pedestrian traffic. Thus, the site offers both an example of applying this technique to non-desert terrain and the ability to use this technique on soil that has not lain undis- 

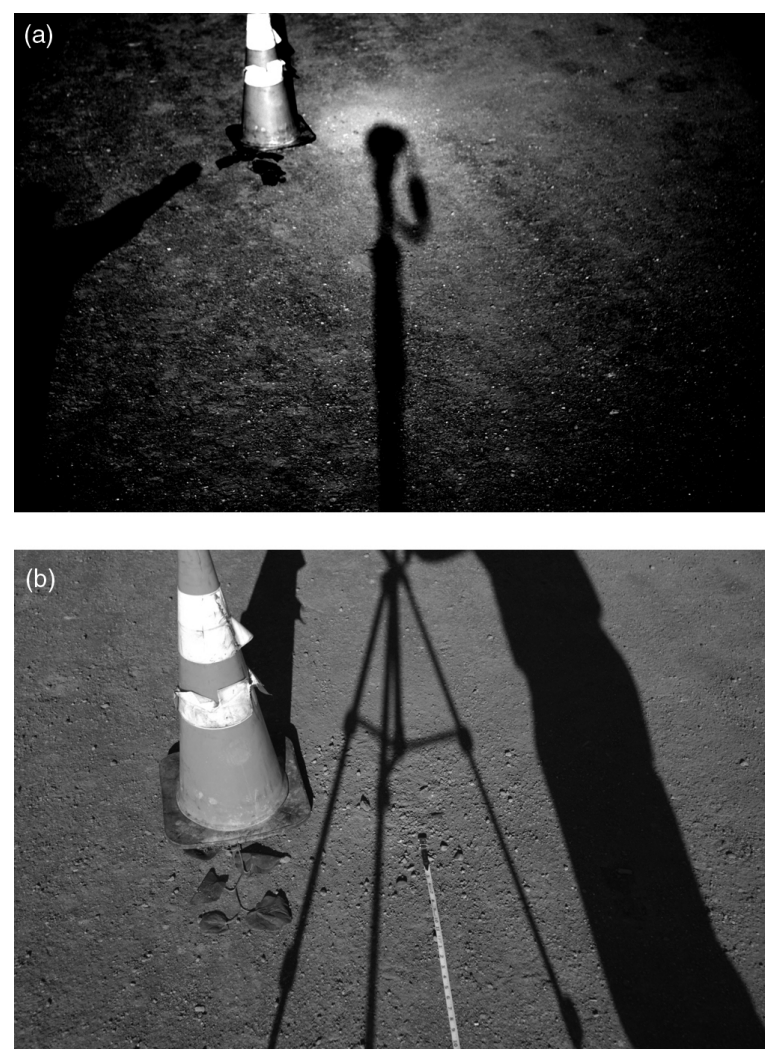

Figure 5. (a) Illumination surge near $0^{\circ}$ phase angle associated with disturbed soil seen in contrast-stretched image. The shadow of the camera body is in the lower right of the patch of disturbed soil. (b) Same disturbed region of ground seen at an angle of $50^{\circ}$ from nadir and high phase angle to simulate a more typical viewing geometry.

turbed for an exceedingly long period. The measurement used passive solar illumination, which is collimated to $0.5^{\circ}$. Several holes were dug and refilled in early morning and allowed to dry throughout a clear summer day. Upon return in the late afternoon, one remained untraversed and it was immediately apparent that macroscopic shadowing dominated any granular photometric effect making the patch of disturbed soil obvious to the unaided eye. In order to present a realistic and difficult simulation of detecting the location of a buried device we disguised the site by smoothing the disturbed patch to eliminate shadows and by sprinkling dirt gathered from the top surface of nearby lesser-disturbed terrain to match albedos. We completed the disguise by randomizing any patterns by lightly brushing the surface with leafy vegetation. The resulting disturbed patch of soil is indeed difficult to discern from the lesser disturbed surrounding terrain under usual viewing geometries 
(Figure 5b). However, when the same disturbed spot is imaged at low phase angle (with the sun behind the camera), it is readily apparent (Figure 5a). The illumination enhancement is not centered on the camera and thus is not glory or another effect only associated with low phase angle, but is associated with the patch of disturbed soil. The lowest phase angle achieved is about $0.5^{\circ}$ because the zero degree phase angle is blocked by the shadow of the camera. Comparison of the brightness of the disturbed patch with a patch of lesserdisturbed soil at an equivalent phase shows the surge is approximately $4 \%$. This effect is detected at $>0^{\circ}$ phase angle, using not completely collimated illumination reflected from a patch of soil disguised to the unaided eye in a background of soil that was not pristine. The opposition effect raised the reflectance of the soil significantly compared to observations at larger phase angles. The disturbed soil at $0.5^{\circ}$ phase angle is $17 \%$ brighter than the lesserdisturbed road surface at a greater phase angle and outside the range of the opposition surge (Figure 6). As the phase angle of the disturbed soil exceeds $1.5^{\circ}$, its brightness decreases to nearly $6 \%$ greater than the background road surface. The existence of some backscattered illumination enhancement to approximately $1.5^{\circ}$ is consistent with a low-albedo soil (relative to quartz, etc). Backscattering in higher-albedo desert soils may be stronger, especially using better collimated illumination than sunlight and at lower phase angle. The approximately $6 \%$ higher reflectivity of the disturbed patch compared to the background road surface demonstrates that disturbing moderate albedo soil will also brighten them, consistent with the previous section.

\section{Discussion and Conclusions}

The three optical methods presented here for detecting disturbed soils to infer the presence of buried mines each have strengths and weaknesses. The existing TIR spectral method is well-proven in deserts and other terrains dominated by silicate lithology. However, that technique would not work well to detect mines buried in carbonate-dominated material. Instead, laboratory measurements suggest that increased reflectance in the SWIR associated with finer-grained material could be used to detect that a surface has been disturbed independent of lithology. The technique appears to work as well as the TIR spectral method for a silicate particulate surface and is even more effective for carbonates. However, only the $2.4 \mu \mathrm{m}$ region appears suitable because the other $; 2.5-\mu \mathrm{m}$ portion of the spectrum are affected by absorption features which could be confused with a grain-size induced reflectance difference. Additionally, while the 3-4- $\mu \mathrm{m}$ region is also devoid of absorption features, other than the $\mathrm{C}-\mathrm{H}$ stretch from 3.2 to $3.5 \mu \mathrm{m}$, spectral contrast is expected to be low at those wavelengths due to the presence of some thermal emission 


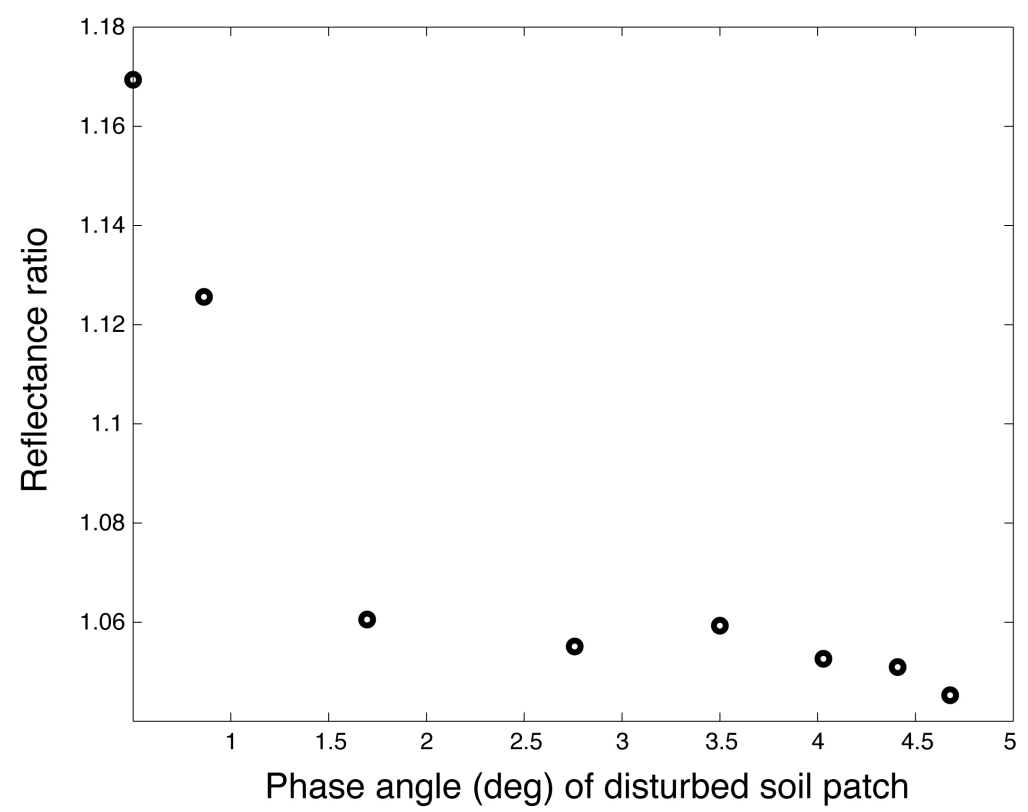

Figure 6. Ratio of the reflectance within pixels of the disturbed soil to pixels about $2^{\circ}$ to the right in soil of the background road. In the first datum, pixels of disturbed soil at $0.5^{\circ}$ are selected. They are about $17 \%$ brighter than the background road at a higher phase angle. The remaining 7 data points are ratios of the same portion of the disturbed soil but from observations at increasing phase angles. The same portion of the disturbed region that is at $0.5^{\circ}$ phase in the first observation is near $1.6^{\circ}$ in the second observation and so on. At the minimum phase angle of the disturbed soil in the second observation, the disturbed patch is only $6 \%$ brighter than the background road that is at a $2^{\circ}$ higher phase angle.

and because the atmosphere is also less transparent than at shorter wavelengths. Additionally, the reliance on an albedo difference risks introducing error if there is a reflectance difference in the subsurface material that is now on the surface. Fieldwork needs to be conducted to test the viability of this technique. The third technique proposed, photometric backscattering, appears to offer a promising new means for characterizing the relative grain size of a surface. Laboratory, theoretical, and preliminary field measurements consistently suggest that photometric backscattering can be used to infer relative grain size. The effect in bright media such as desert soils should be 10s of percent at visible and NIR wavelengths and should work with a wide range of compositions, will however strongly depend on the albedo. As with relying on SWIR reflectance to infer disturbance, the possibility for error exists from albedo changes that could be confused with or reduce the effect of backscattering. It may prove prudent to conduct backscattering measurements at SWIR wavelengths to also take advantage of the wavelength-dependent 
reflectance increase associated with finer-grained materials.

\section{Acknowledgements}

Thanks to Dr. James Bauer for insightful discussion and initial suggestion to investigate the opposition surge effect. Thanks to Dr. Bonnie Buratti for the unpublished data of powdered sandstones. Assistance in the field by Amit Mushkin and discussions with Dr. Alan Gillespie also proved essential. Much of this initial was funded by a small portion of a federal contract to the Planetary Science Institute and subsequently by overhead funds from the Johns Hopkins University Applied Physics Laboratory.

\section{References}

Adams, J.B. (1975) Interpretation of visible and near-infrared diffuse reflectance spectra of pyroxenes and other rock-forming minerals, In Infrared and Raman Spectroscopy of Lunar and Terrestrial Minerals, Academic Press, New York, 94-116. Buratti, B. J., J. K. Hillier, M. Wang (1996) The Lunar opposition surge: observations by Clementine, Icarus, 124, p. 490. Conel, (1969) Estep-Barnes, P.A. (1977) Infrared spectroscopy, in Physical Methods, In J. Zussman (eds.) Determinative Mineralogy, pp. 529-603. Academic, New York. Hapke, B. W. (1986) Bidirectional reflectance spectroscopy IV. The extinction coefficient and the opposition effect, Icarus 67, 264-280. Johnson, J. R., P. G. Lucey, K. A. Horton, E. M. Winter (1998) Infrared measurements of pristine and disturbed soils 1 . Spectral contrast differences between field and laboratory data, Remote Sensing of Environment, 64, p. 34. Mishchenko, M. I., J-M. Luck, and T. M. Nieuwenhuizen (2000) Full angular profile of the coherent polarization opposition effect, J. Opt. Soc. Am., 17, p.888. Muinonen, K., K. Sarrinen (2000) Ray optics approximation for Gaussian random cylinders, J. Quantitative Spectroscopy and Radiative Transfer, 64, p. 201. Nelson, R. M., Hapke, B.W., Smyth, W.D., Horn, L.J. (1998) Phase Curves of Selected Particulate Materials: The Contribution of Coherent Backscattering to the Opposition Surge, it Icarus, 131, 223-230. Okin, G. S. and T. H. Painter (2004) Effect of grain size on remotely sensed spectral reflectance of sandy desert surfaces, Remote Sensing of Environment, 89, p. 272. Rupp, R. R., Z. Derzko, and G. R. Ax, Jr. (2004) Lightweight airborne standoff minefield detection laser prototype, Proc. SPIE, Vol. 5415, p. 1151. Salisbury, J. (1987) Mid-Infrared (2.1-25 micron) spectra of minerals, USGS Open File Report 87-263. Salisbury, J. and A. Wald (1992) The role of volume scattering in reducing spectral contrast of reststrahlen bands in spectra of powdered minerals, Icarus, 96, 121-128. Shkurotov, Y. G., M. A. Kreslavasky, A. A. Ovcharenko, D. G. Stankevich, and E. S. Zubko (1999) Opposition effect from Clementine data and mechanisms of backscatter, Icarus, 141, p. 132. 